\title{
GPPS-BJ-2019-104
}

\section{IMAGING DIAGNOSITICS OF COMBUSTION INSTABILITY IN PREMIXED SWIRLING COMBUSTION}

\author{
Yao Yang \\ School of Aeronautics and Astronautics, \\ Zhejiang University \\ youth_y@zju.edu.cn \\ Hangzhou, China
}

\author{
Gaofeng Wang \\ School of Aeronautics and Astronautics, \\ Zhejiang University \\ gfwang@zju.edu.cn \\ Hangzhou, China
}

\author{
Yuanqi Fang \\ School of Aeronautics and \\ Astronautics, Zhejiang \\ University \\ yqfang@zju.edu.cn \\ Hangzhou, China
}

\author{
Yifan Xia \\ School of Aeronautics and \\ Astronautics, Zhejiang \\ University \\ xiayifan@zju.edu.cn \\ Hangzhou, China
}

\author{
Liang Zhong \\ School of Aeronautics and \\ Astronautics, Zhejiang \\ University \\ liangzhong@zju.edu.cn \\ Hangzhou, China
}

\begin{abstract}
An experimental study on combustion instability is presented with focus on propane-air premixed swirling flames. Swirling flames under self-excited oscillation are studied by imaging of visible light and $\mathrm{OH}^{*}$ chemiluminescence filter under several typical conditions. The dynamical characteristics of swirling flames were analysed by Dynamic Mode Decomposition (DMD) method. Three types of unstable modes in the combustor system were observed, which correspond to typical acoustic resonant modes (LF mode, $\mathrm{C}_{1 / 4}$ mode and $\mathrm{P}_{1 / 2}$ mode) of the combustor system. The combustion instability is in the longitudinal mode. Furthermore, the structure of downstream hot burnt gas under stable combustion and unstable combustion is studied by imaging of visible light and near-infrared light. Results show that there is a significant difference in the downstream flow under stable combustion and unstable combustion. The DMD spectrum of the flame and the downstream hot burnt gas obtained is the same, which is close to the characteristic frequency of acoustic pressure captured by the microphone signal. The visible light and near-infrared light imaging observation method adopted in this paper provides a new imaging method for the investigation of thermo-acoustic instability.
\end{abstract}

\section{INTRODUCTION}

Thermo-acoustic instabilities are frequently observed in various combustion systems, including aero engines and gas turbines. Thermo-acoustic instabilities occur when the heat release and acoustic pressure are in phase. Heat release rate fluctuations give rise to pressure fluctuations and reversely pressure fluctuations influence the unsteady heat release. Thermo-acoustic instabilities are also called combustion oscillation. The Rayleigh criterion states that combustion oscillation occurs when the heat release pulsation of the flame provides more energy to the sound field than the inherent acoustic dissipation of the system. When combustion oscillation occurs, huge noise and system resonance will happen, which may do harm to the life of the combustion system. Studies by Schuller [2003], Huang, [2004], Weigand [2006] and Culick [2006] show that there are many factors that may induce combustion oscillations, including vortex shedding, swirling, inlet temperature, insufficient gas supply and flow fluctuation. For premixed combustion, the vortex can not only influence the flame front, but also generate noise and interact with the combustion process. Selle [2006] shows that combustion oscillations usually occur usually in the corresponding acoustic mode of the combustion chamber, but also sometimes a coupled mode of acoustic and gas convection studied by Pankiewitz [2003].

It is well known that swirling flames are quite sensitive to acoustic waves. The interaction between heat release and acoustic waves might lead to the self-sustained thermoacoustic oscillations. There are many experimental and numerical investigations about combustion oscillations of swirling flames. Palies et al [2010] studied the flame dynamics under external-force excitation by using ICCD, which states unsteady heat release is due to the velocity pulsation. Li et al [2015] investigated the effect of acoustic perturbation on the hear release pulsation. Kim et al[2009] studied the dynamics of flame by $\mathrm{OH}^{*}, \mathrm{CH}^{*}$ and $\mathrm{CO}_{2} *$ 
chemiluminescence for different synthesis gas. Meier et al [2010] studied the reaction zone structure of the swirling flame by using $\mathrm{OH}^{*}$ PLIF and PIV techniques, and pointed out that the Proceeding Vortex Core influenced the unstable combustion mode. Min et al [2015] used high-speed $\mathrm{OH}^{*}$ chemiluminescence intensity of different synthesis gas, and obtained instability mode by using Proper Orthogonal Decomposition. Huang et al. [2012] studied the combustion dynamics in a premixed swirl-stabilized combustor with numerical and showed that the inlet temperature influences the stability characteristic. Guo et al [2009] studied the flame structure of the lean premixed swirling flame and pointed out that coupling between the flow pulsation and the heat release pulsation was the main reason for combustion instability.

Generally, the swirling flames are the main focus during previous investigations. The behaviour of the downstream hot gas is rarely studied. However, in aero-engines, the hot burnt gases from the combustion chamber have important effects to the downstream turbine vanes, which may induce hot spot. Thus, diagnostic studies on the downstream hot gas of the swirling flame is interesting and also with practical value. In the present work, the characteristic modes of unstable combustion are experimentally investigated. Especially, the features of the downstream hot burnt gas under unstable combustion are discussed.

This paper is structured as follows. The experimental configuration and condition are briefly described in Section 2. Experimental results are presented and discussed in Section 3. Finally, the conclusions are drawn in the last section.

\section{EXPERIMENTAL CONFIGURATIONS}

The single-burner combustor is displayed in Fig.1. The combustor is made of plenum, swirling injector and combustion chamber. The propane and air are delivered through a Venturi-tube to perfectly mixed and then conveyed into the plenum by four supplying pipes to avoid equivalence ratio fluctuations. The premixed mixtures flow through a set of grids to obtain a roughly uniform distribution of axial velocity over the whole cross-section. The convergent nozzle takes the similar role as the girds. Finally, the premixed gas is fed to the combustion chamber through a swirling injector. The parameter of the swirling injector is shown in Fig.2. The diameter of the swirling injector tube is $10 \mathrm{~mm}$, and the swirl number is 0.82 . The combustion chamber is made of cylindrical quartz tube with $50 \mathrm{~mm}$ inner diameter and 200 $\mathrm{mm}$ height. The sequences are recorded by fast speed camera PCO dimax-HD and Phantom M110. The frame rate and shutter time were set at $2000 \mathrm{~Hz}$ and $500 \mu$ s for both cameras, respectively. A UV-IR-cut filter $(400-680 \mathrm{~nm})$ is adopted on one lens to capture chemiluminesence intensity from the $\mathrm{CH}^{*}, \mathrm{C}_{2}$ *and $\mathrm{CO}_{2} *$ in the flame fronts. A Near-IRpass filter $(780-2000 \mathrm{~nm})$ is mounted on the other camera's lens to record the hot burnt gas on the downstream of swirling flame. The Photomultiplier (PMT), equipped with $293-323 \mathrm{~nm}$ filter, is located at a distance of $500 \mathrm{~mm}$ from the swirling burner axis. The PMT is used to record the $\mathrm{OH}^{*}$ chemiluminescence intensity. It has been demonstrated that the intensity of $\mathrm{OH}^{*}$ signal is nearly proportional to heat release rate by Schuller [2002]. Two microphone sensors are adopted to record the acoustic pressure signals in both combustion chamber and plenum. The positions of microphone sensors are presented in Fig.1, One is $20 \mathrm{~mm}$ far from the bottom of the swirling injector, the other is $150 \mathrm{~mm}$ away from the swirling injector outlet plane. Simultaneous acquisitions of all instruments are controlled by DG535 in the experiment.

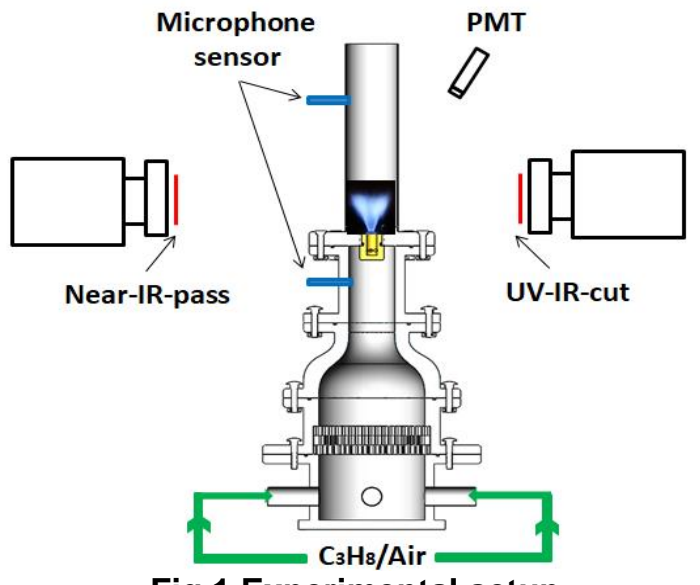

Fig.1 Experimental setup

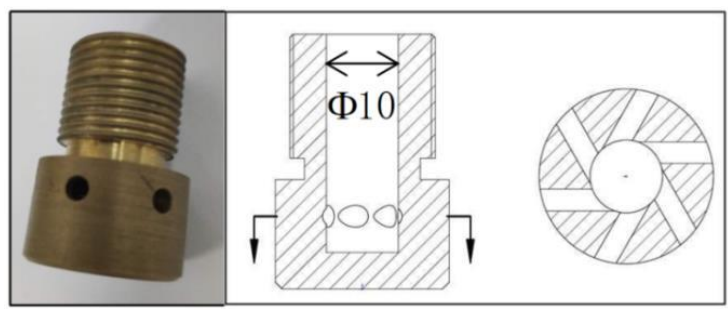

Fig.2 Swirling injector

All the experiments are carried out at ambient conditions ( $T=293 \mathrm{~K}, P_{0}=1$ bar), and all the measurements presented in this study are carried out in steady thermal state.

Table 1

Summary of experiment conditions

\begin{tabular}{lcc}
\hline & $V_{f} / L / \min$ & $\Phi$ \\
\hline Case 1 & & 1.00 \\
Case 2 & 0.7 & 1.05 \\
Case 3 & & 1.15 \\
\hline Case 4 & 1.20 \\
Case 5 & \multirow{2}{*}{1.0} & 1.15 \\
Case 6 & & 1.00 \\
\hline
\end{tabular}

The working conditions of six chosen cases are listed in Tab.1, which are also marked out in Fig.3. All chosen cases are in unstable combustion except for Case 6.

\section{EXPERIMENTAL RESULTS AND DISCUSSION 3.1 STABILITY MAP}

The Reynolds number of experiments ranges from $R e=$ 1543 to 3666 , which corresponds to the bulk velocity ranging from $U_{b}=2.32$ to $5.52 \mathrm{~m} / \mathrm{s}$. The stability map is plotted in Fig.4. It can be observed that unstable combustion occurs in 
rich-fuel zone ( $\Phi$ equivalence is over 1.0). Three types of combustion instability were found in the experiments, which are named as Type-1, Type- 2 and Type- 3 , respectively. The partition of the three types is based on the frequency of the unstable mode.

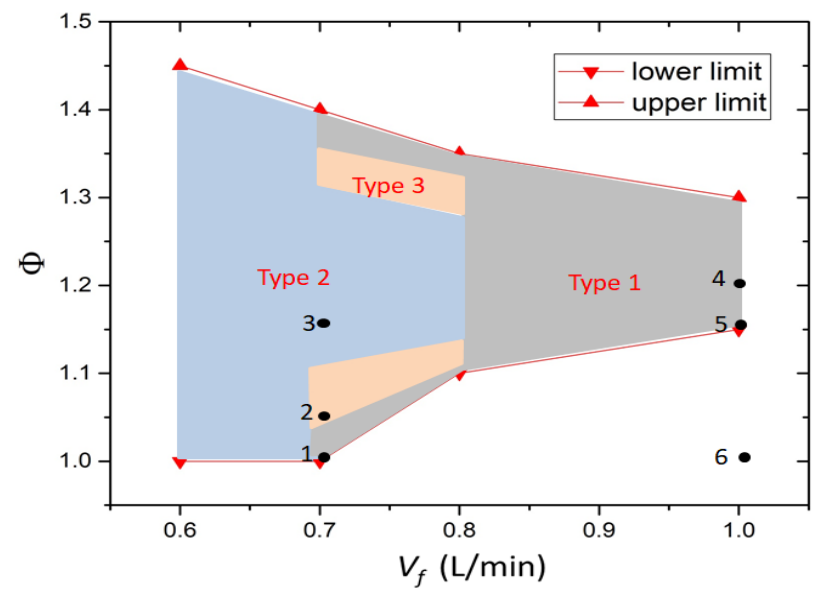

Fig.3 Stability map (the black spot represents the six cases chosen)

As the flow rate of fuel decreases, combustion instability occurs in a wider range. When the flow rate of propane is in the range of $V_{f}=0.8 \sim 1.0 \mathrm{~L} / \mathrm{min}$, unstable combustion is in Type-1. When the flow rate of propane is in the range of $V_{f}=0.6 \sim 0.7 \mathrm{~L} / \mathrm{min}$, unstable combustion is in Type-2. When the flow rate of propane is in the range of $V_{f}=$ $0.7 \sim 0.8 \mathrm{~L} / \mathrm{min}$, these three types of combustion instability may occur as the equivalence ratio changes.

\subsection{UNSTABLE MODES}

The combustor in present work shown in Fig.4 is a typical three-duct system. According to the acoustic analysis, there may be several typical acoustic resonant modes in this kind of combustor. When the coupled factor $\theta=\frac{s_{2}}{s_{3}} \frac{\rho_{3}}{\rho_{2}} \frac{c_{3}}{c_{2}}$ becomes small, the mode of the combustor chamber and the injector tube could be decoupled and analysed separately[26]. The plenum and the injector tube can be seen as a resonator. This corresponding mode is named as LF mode (Low Frequency mode) in the present work. Due to the closed inlet and open outlet boundary conditions, a quarterwave $\left(\mathrm{C}_{1 / 4}\right)$ mode and three-quarter $\left(\mathrm{C}_{3 / 4}\right)$ wave mode exist in the acoustic mode of present combustor system. Similarly, the boundary condition for plenum is closed inlet and closed outlet, respectively. Thus, half-wave mode $\left(\mathrm{P}_{1 / 2}\right)$ and fullwave $\left(\mathrm{P}_{2 / 2}\right)$ mode exist. Theoretically, high-order mode may also exist in plenum $\left(\mathrm{P}_{3 / 2}, \mathrm{P}_{4 / 2}\right.$ modes etc. $)$

The DMD method developed by Schmid [2010] was adopted to study the snapshot sequences taken by the high speed camera with UV-IR-cut filter. The frame rate of the camera is $2000 \mathrm{~Hz}$. Meanwhile, one thousand consecutive sequences were adopted for DMD analysis. The DMD spectrums for Case 1, Case 2 and Case 3 are shown in Fig.5. Meanwhile, Fig.6 and Fig.7 shows the PSD spectrums of $\mathrm{OH}^{*}$ chemiluminesence intensity and acoustic pressure, respectively. The $\mathrm{OH}^{*}$ chemiluminesence intensity is obtained by PMT. The acoustic pressure is obtained by two microphone sensors, shown in Fig.1.

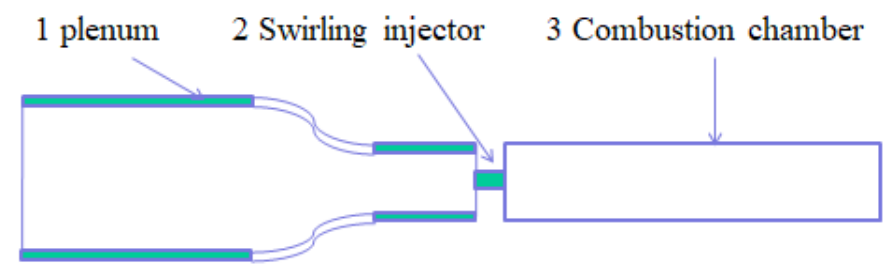

\section{Fig.4 Schematic illustration of three-duct configuration}

For Case 1, the intrinsic frequency of DMD spectrums is $38 \mathrm{~Hz}$, and there are two main intrinsic frequencies $(38 \mathrm{~Hz}$ and $674 \mathrm{~Hz}$ ) occur in the acoustic signal of plenum and no intrinsic frequency in the acoustic signal of combustion chamber, which proves that the mode of $38 \mathrm{~Hz}$ is the resonant mode of plenum and injector. Thus, the mode of 38 $\mathrm{Hz}$ is corresponding to LF (Low Frequency) mode. Besides, $76 \mathrm{~Hz}$ and $114 \mathrm{~Hz}$ also can be observed in DMD spectra of flame for case 1 . These intrinsic frequencies are the double and triple frequency of the $38 \mathrm{~Hz}$, which could be regarded as double-frequency resonant mode. The amplitude of double-frequency resonant mode is weaker than the basic intrinsic frequency. The LF mode is a typical resonant mode for the plenum resonator. It is also found in the experiments that combustion instability in the LF mode can occur without combustion chamber, which proves that LF mode is a coupled mode of injector tube and plenum instead of combustion chamber.

For Case 3, the DMD spectrums show one intrinsic frequency $(272 \mathrm{~Hz})$. Meanwhile, this frequency is observed in the acoustic signals of the combustion chamber, as well as the plenum. This frequency is also observed in the PSD spectrums of $\mathrm{OH}^{*}$ signal. Obviously, this is the resonant mode $\left(\mathrm{C}_{1 / 4}\right.$ mode $)$ corresponding to the whole combustor system. Similarly, a double-frequency resonant mode (545 $\mathrm{Hz}$ ) is observed in both the DMD spectrums of flame and the PSD spectrums of $\mathrm{OH}^{*}$ signal.

For Case 2, two intrinsic frequencies $(51 \mathrm{~Hz}$ and 275 $\mathrm{Hz}$ ) are observed in the DMD spectrums. They are also found in the PSD spectrums of $\mathrm{OH}^{*}$ signal. Meanwhile, these two main intrinsic frequencies also exist in the acoustic signal of plenum. But, only one intrinsic frequency ( 275 $\mathrm{Hz}$ ) is presented in the acoustic signal of combustor chamber. In other word, this unstable mode is mixed by LF mode and $\mathrm{C}_{1 / 4}$ mode.

Interestingly, one special intrinsic frequency exists in the acoustic signal of plenum for all three cases, $(674 \mathrm{~Hz}$ for Case 1, $682 \mathrm{~Hz}$ for Case 2 and $681 \mathrm{~Hz}$ for Case 3). This frequency is in absence of the acoustic signal in combustor chamber. This is a resonant mode in plenum only, which is corresponding to the $\mathrm{P}_{1 / 2}$ mode. Though, this mode is weak. It can be found in the PSD spectrums of $\mathrm{OH}^{*}$ signal indeed. But it is not detected in the DMD spectrums of flame. The reason for this phenomenon is as following: The frame rate of imaging is $2000 \mathrm{~Hz}$, which is smaller than the sampling 
rate of $\mathrm{OH}^{*}$ signal and acoustic signal. It might be not enough for DMD method to distinguish such a weak and relatively high frequency $\mathrm{P}_{1 / 2}$ mode due to the noise effects, such as turbulence.

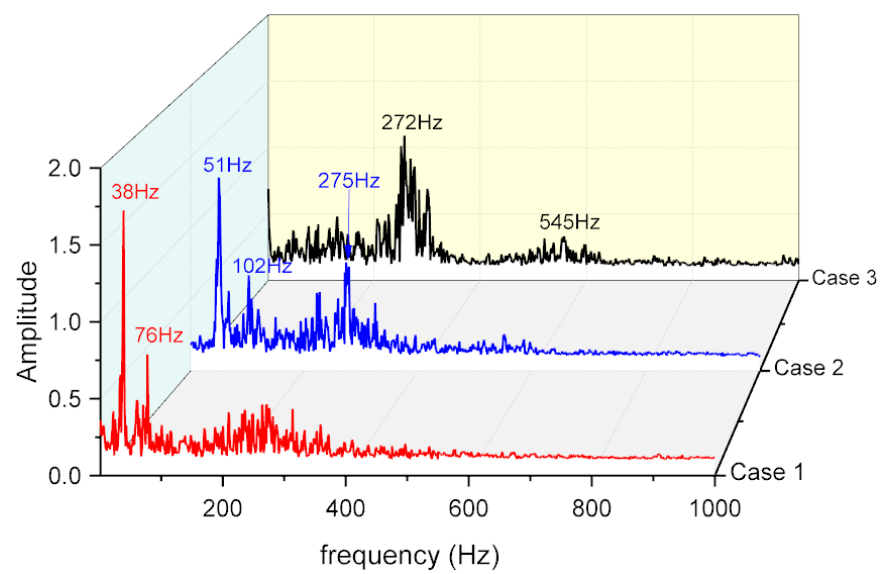

Fig.5 DMD spectrums for flame (the red line is for Case 1, the blue line is for Case 2 and the black line is for Case 3)

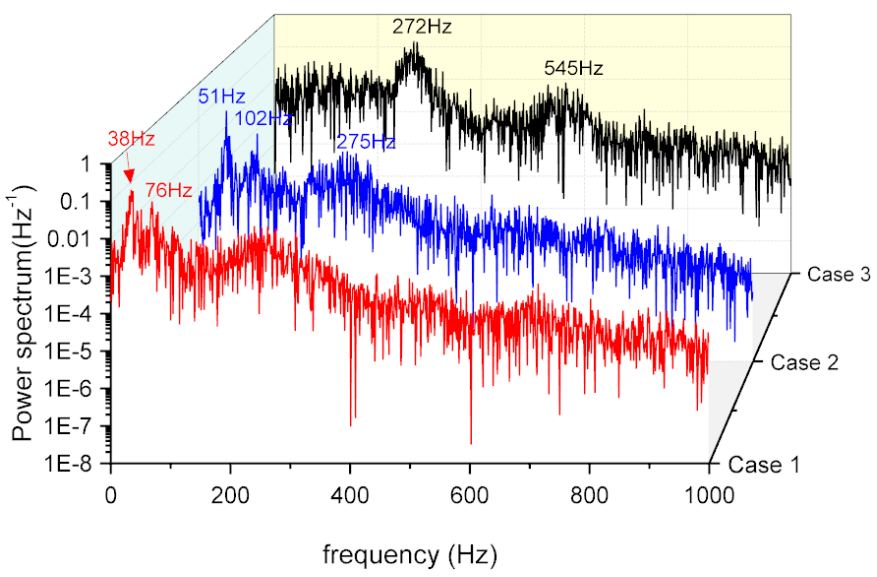

Fig.6 PSD spectrums of $\mathrm{OH}^{\star}$ chemiluminesence intensity

The mode phases of Case 1 and Case 3 are shown in Fig.8 and Fig.9, respectively. It can be observed that combustion instability is in longitudinal mode. For Case 2, both $\mathrm{LF}$ and $\mathrm{C}_{1 / 4}$ modes are observed in Fig.10. Though, both modes are longitudinal, there is difference in the phase map. It can be observed that LF mode presents a process of change from downstream to upstream, while the $C_{1 / 4}$ mode is in an opposite process.

As discussed, Case $1(\Phi=1.0)$ is in LF mode. As the equivalence ration increases, Case $2(\Phi=1.05)$ is in a mixed mode of $\mathrm{LF}$ mode and $\mathrm{C}_{1 / 4}$ mode. The amplitude of LF mode in Case 2 is weaker than that in Case 1. When the equivalence ratio increases to Case $3(\Phi=1.10)$, the $\mathrm{LF}$ mode disappeared and $\mathrm{C}_{1 / 4}$ mode is the dominant mode. Recalling the stability map given in Fig.3, Type-1 is a region where LF mode occurs; Type- 2 is a region in which $\mathrm{C}_{1 / 4}$ mode occurs; and Type- 3 is a region belonging to transition mode between Type- 1 and Type-2. Meanwhile, it can be observed that LF mode occurs in the region of relatively high combustion power. $\mathrm{C}_{1 / 4}$ mode occurs in the region of relatively low combustion power. Mixed mode is more likely to a transition mode between $\mathrm{LF}$ mode and $\mathrm{C}_{1 / 4}$ mode.

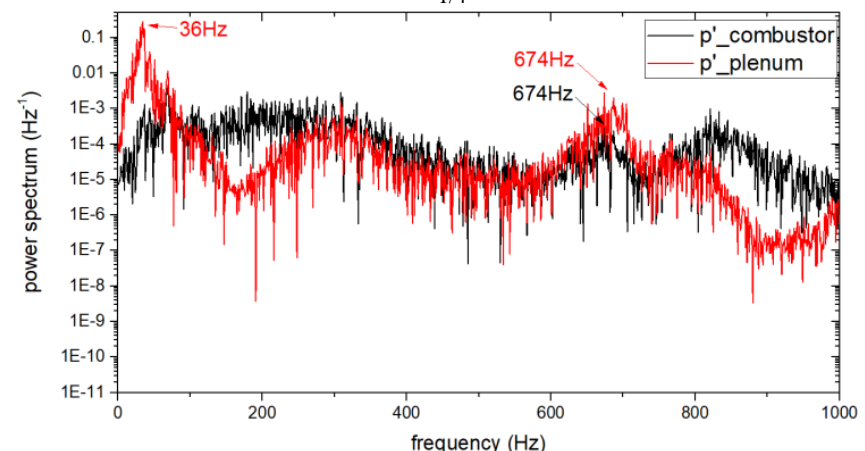

(a) Case 1

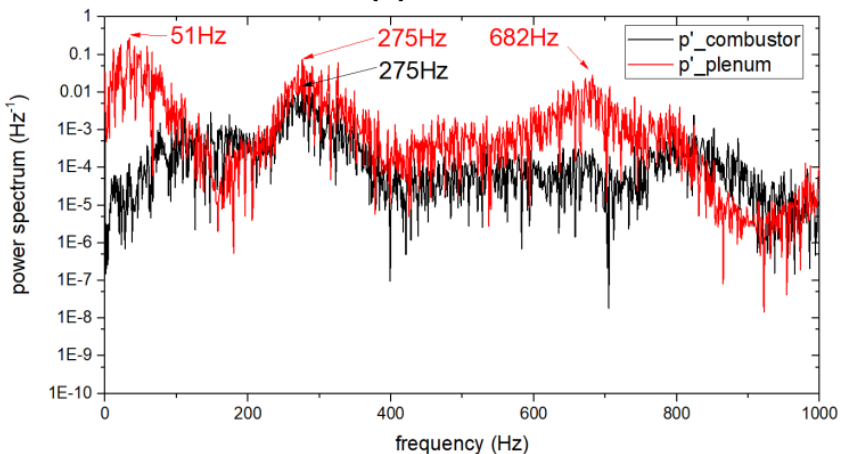

(b) Case 2

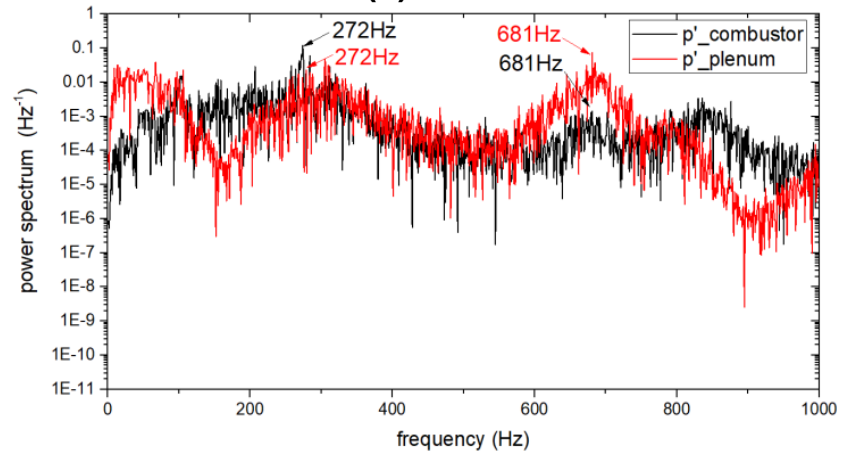

(c) Case 3

Fig.7 PSD spectrums of the acoustic pressure in combustion chamber and plenum

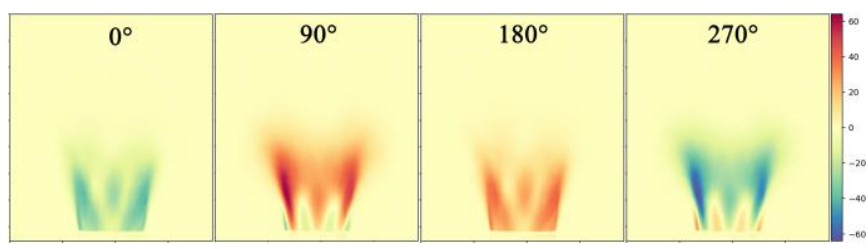

Fig.8 DMD mode $(f=38 \mathrm{~Hz})$ for Case 1

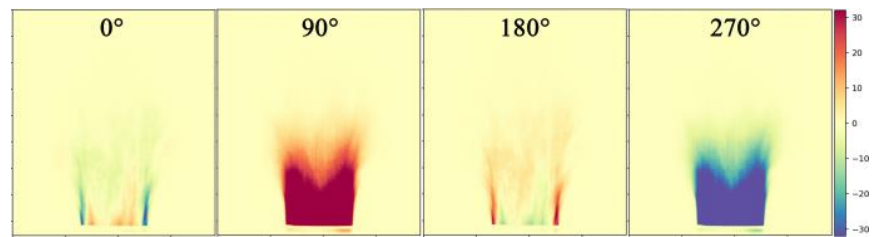

Fig. 9 DMD mode $(f=272 \mathrm{~Hz})$ for Case 3 


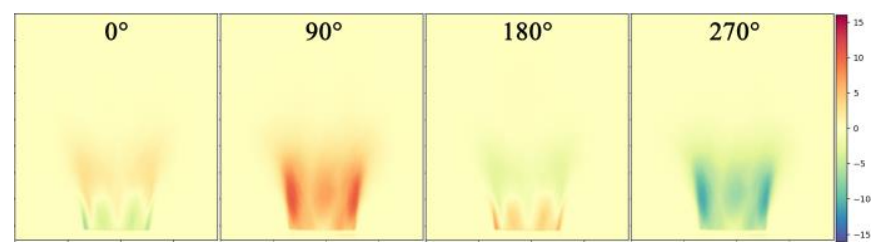

(a) DMD mode $(f=51 \mathrm{~Hz})$

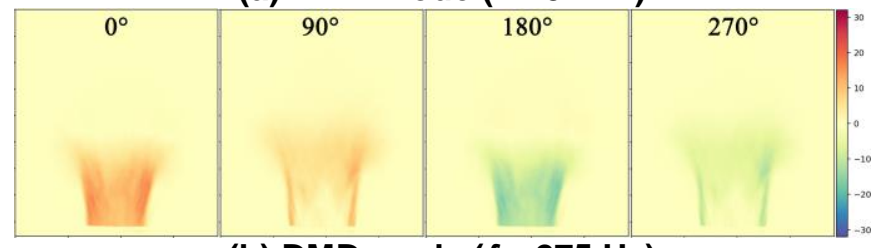

(b) DMD mode $(f=275 \mathrm{~Hz})$

Fig.10 DMD mode for Case 2

The unstable modes and intrinsic frequencies of all cases are listed in Tab.2. The intrinsic frequency corresponding to LF mode varies from $32 \mathrm{~Hz}$ to $51 \mathrm{~Hz}$ in all cases studied. The intrinsic frequency corresponding to $\mathrm{C}_{1 / 4}$ mode for two cases is almost the same. The intrinsic frequency corresponding to $\mathrm{P}_{1 / 2}$ mode for three cases (Case 1, Case 2, and Case 3) is also in a narrow band. There is a little shift in the frequency for each resonant mode. It may be explained as following. The adiabatic flame temperature of cases in each resonant mode is roughly close. When the equivalence ratio changes, the flow rate of air is change. This leads to changing of temperature in combustion chamber wall, thereby influencing the intrinsic frequency of thermoacoustic instability. Because the experiments are operated under rich-fuel situations, the change of temperature is mainly due to the flow rate of air. Actually, the effect of wall temperature on unstable intrinsic frequency is quite complex. These discussions may be further investigated by the thermoacoustic model with Flame transfer function in future works.

Table 2

\section{Comparison of experimental results}

\begin{tabular}{lcc}
\hline & Resonant mode & Experimental results \\
\hline Case 1 & & $38 \mathrm{~Hz}$ \\
Case 2 & \multirow{2}{*}{ LF mode } & $51 \mathrm{~Hz}$ \\
Case 4 & & $46 \mathrm{~Hz}$ \\
Case 5 & & $32 \mathrm{~Hz}$ \\
\hline Case 2 & \multirow{2}{*}{$\mathrm{C}_{1 / 4}$ mode } & $275 \mathrm{~Hz}$ \\
Case 3 & & $272 \mathrm{~Hz}$ \\
\hline Case 1 & & $674 \mathrm{~Hz}$ \\
Case 2 & $\mathrm{P}_{1 / 2}$ mode & $682 \mathrm{~Hz}$ \\
Case 3 & & $681 \mathrm{~Hz}$ \\
\hline
\end{tabular}

\subsection{DIAGNOSIS OF DOWNSTREAM HOT BURNT GAS}

In order to study the effect of the downstream flow field on the swirling flame, simultaneous imaging acquisition of swirling flame and downstream hot burnt gas was adopted.

The consecutive imaging of flame and hot burnt gas for Case 6 is shown in Fig.11. Combustion for Case 6 is in steady situation. The V-shape flame is clear. Meanwhile, the structure of hot burnt gas is also in V-shape. There is no local peak region of heat release in hot burnt gas, which means that the heat release distribution is relatively uniform. The sequences of flame and hot burnt gas of Case 5 are presented in Fig.12. Case 5 is in unstable situation. The flame shape changes greatly in unstable combustion compared to stable one.
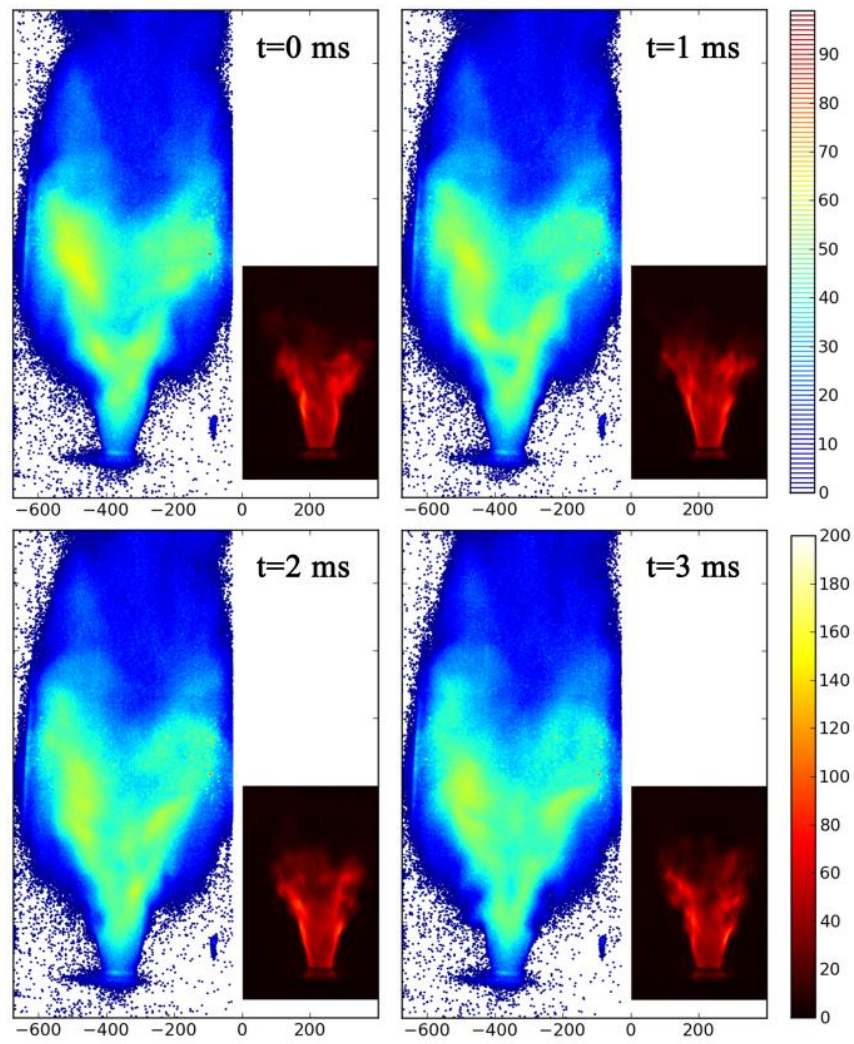

Fig.11 Imaging of flame and hot burnt gas for Case 6(The left of each picture is hot burnt gas, the right one is flame; The above colorbar is for hot burnt gas, The bottom colorbar is for flame)

Although the experimental condition is rich-fuel, there is no flame fragment found in the hot burnt gas. In the imaging sequences, the camera with UV-IR-cut filter could record the visible flame but not the hot burnt gas, which proves that the hot burnt gas is the product of unstable combustion instead of the flame fragment. Compared to the imaging of stable combustion in Case 6, large-scale oscillations are observed in the swirling flame for Case 5. At $\mathrm{t}=8 \mathrm{~ms}$, both the flame and hot burnt gas have strong luminescence intensity, the hot burnt gas is mainly confined to the vicinity of the central axis of the chamber. At $\mathrm{t}=12 \mathrm{~ms}$, the flame shape becomes conical. The flame is nearly quenching because of the insufficient local gas supply caused by velocity fluctuation, and the luminescence intensity of the hot burnt gas is also weakened. At $\mathrm{t}=16 \mathrm{~ms}$, the flame shape changes significantly compared to steady combustion situation. The luminous intensity begins to re-enhance and the heat release is mainly gathered in the upper end of the flame. By imaging of hot burnt gas, it can be observed that a large-scale bot burnt gas mass formed at the downstream of flame. Meanwhile, a knot appears between the flame and hot burnt gas due to sucking. At $\mathrm{t}=17 \mathrm{~ms}$, the luminous intensity of the hot burnt gas mainly occurs in the area where the heat release of flame is larger. The luminous intensity of flame 
and hot burnt gas continues to increase. There is a structure of the outer coil at the upper end of the flame and a nearly broken interface appears at the junction of the flame and hot burnt gas at this moment. At $\mathrm{t}=22 \mathrm{~ms}$, the hot burnt gas develops downstream further. The length of flame becomes longer and the opening angle of flame becomes smaller compared with the structure of previous sequence $(\mathrm{t}=17 \mathrm{~ms})$. At $\mathrm{t}=26 \mathrm{~ms}$, the flame shape becomes relatively uniform with some small-scale irregular structures. When the hot burnt gas is fully developed downstream, a large-scale package of hot burnt gas breaks down. Then, the broken-down package develops downstream and dissipates eventually. After that, the flame is going into the next unstable periodic oscillation.
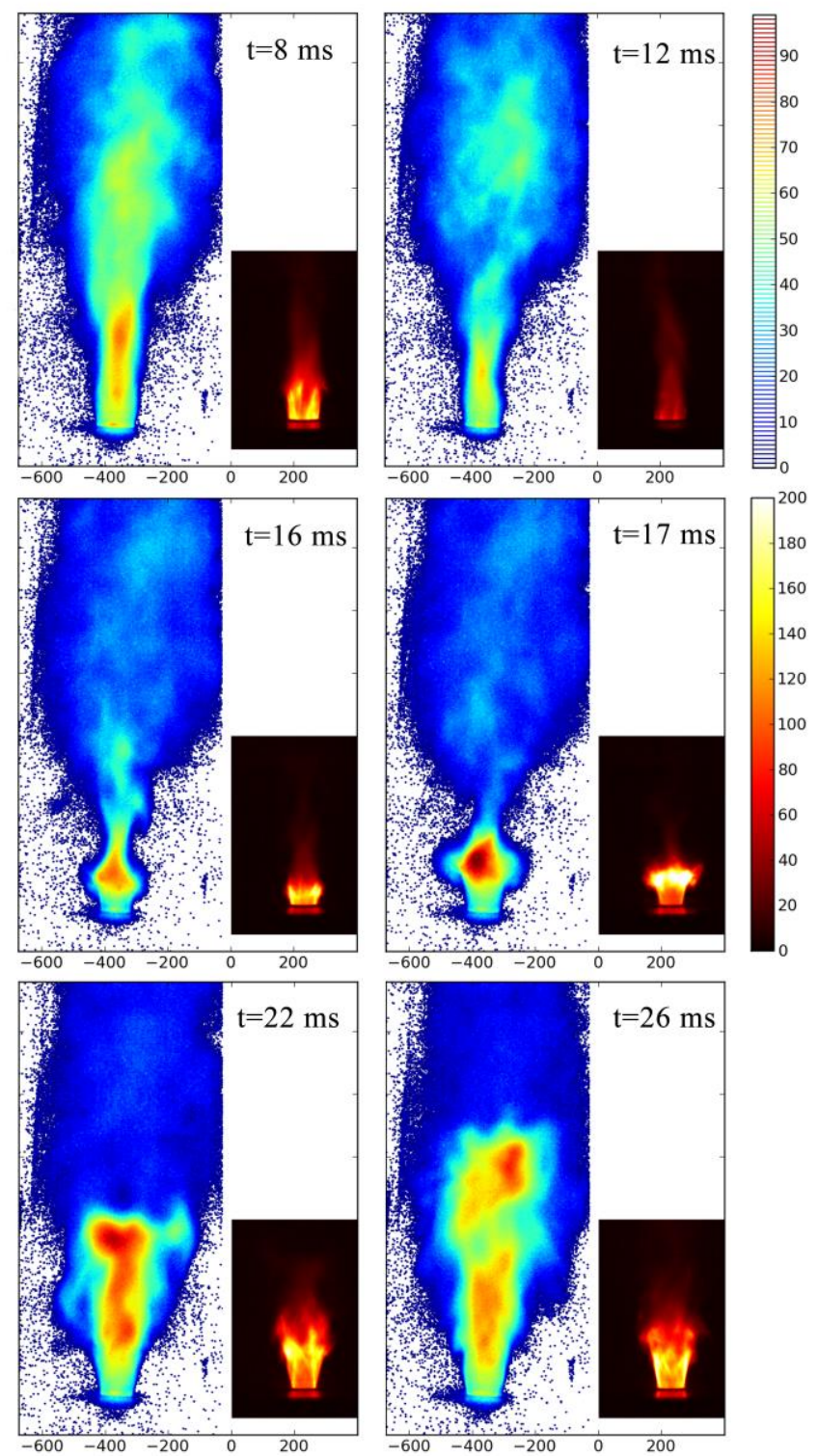

\section{Fig12 Imaging of flame and hot burnt gas for Case 5}

The relationship between normalized integrated luminescence intensity of flame and hot burnt gas for Case 5 is shown in Fig.13. These two signals are within the same frequency. However, there is an apparent time lag in peak point between the flame and hot burnt gas with good repeatability. The peak value of the hot burnt gas signal is always behind the peak value of the flame signal. This can be well explained by the sequences in Fig.12. The hot burnt gas is the burning product, which is at downstream of the flame. It could not be detected by UV-IR-cut imaging. A certain period of time is needed for the package of hot burnt gas generated by the unstable combustion to gather and break down from the flame.

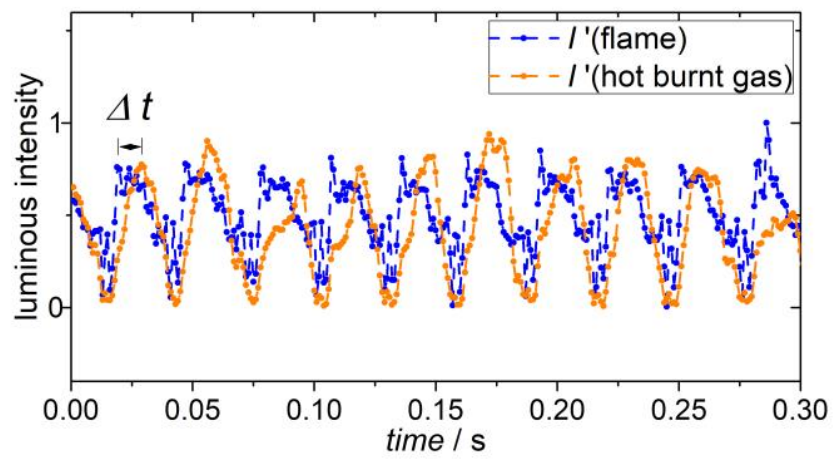

Fig.13 Normalized integrate luminescence intensity of flame and hot burnt gas for Case 5

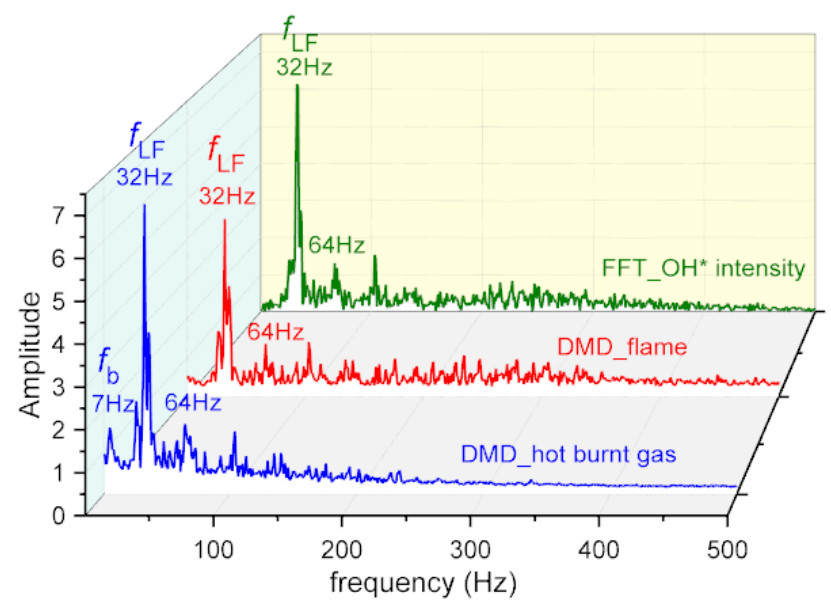

Fig.14 Spectrums of flame, hot burnt gas and $\mathrm{OH}^{\star}$ chemiluminesence intensity for Case 5

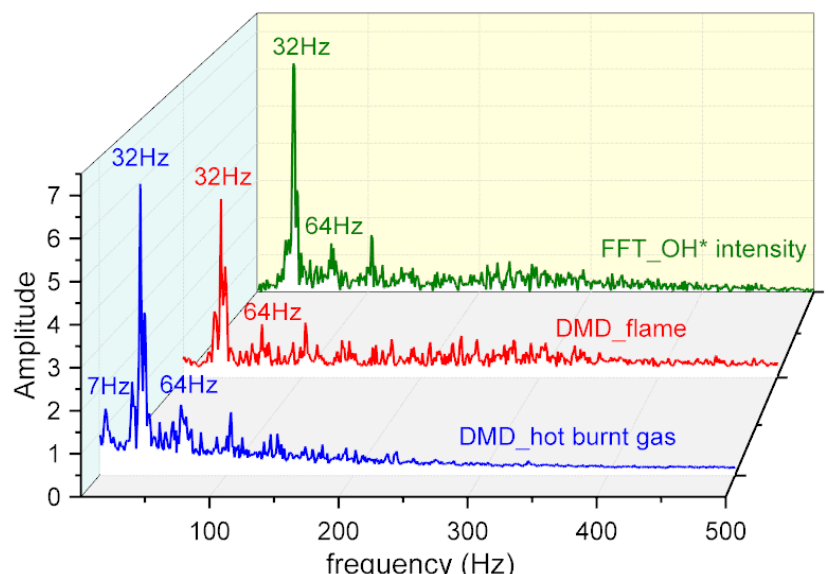

Fig.15 Spectrums of flame, hot burnt gas and $\mathrm{OH}^{\star}$ chemiluminesence intensity for Case 4

The snapshots of flame and hot burnt gas are also analysed by DMD method. The frame rate of camera is 2000 
$\mathrm{Hz}$ and 500 consecutive frames are adopted in Case 4 and Case 5. The DMD spectrums of flame and hot burnt gas for Case 5 are shown in Fig.14, together with the FFT results of $\mathrm{OH}^{*}$ signal. The intrinsic frequency $(32 \mathrm{~Hz})$ is obtained from all three signals for Case 5. Besides, two intrinsic frequencies $(64 \mathrm{~Hz}$ and $96 \mathrm{~Hz}$ ) can be observed in the spectrums of all three signals, which are the double-frequency resonant mode of the basic intrinsic frequency. Similar results can be found in the spectrums for Case 4, shown in Fig. 15. The intrinsic frequency of Case 4 is $46 \mathrm{~Hz}$, which is a little bit higher than that of Case 5. Though, these two cases are in the same unstable mode (LF mode). A little shift of intrinsic frequency may do happen, which have been discussed in previous comments. Apart from the LF mode, a special frequency $\left(f_{\mathrm{b}}\right)$ is found only in the spectrums of hot burnt gas, which is not detected in spectrums of other signals.
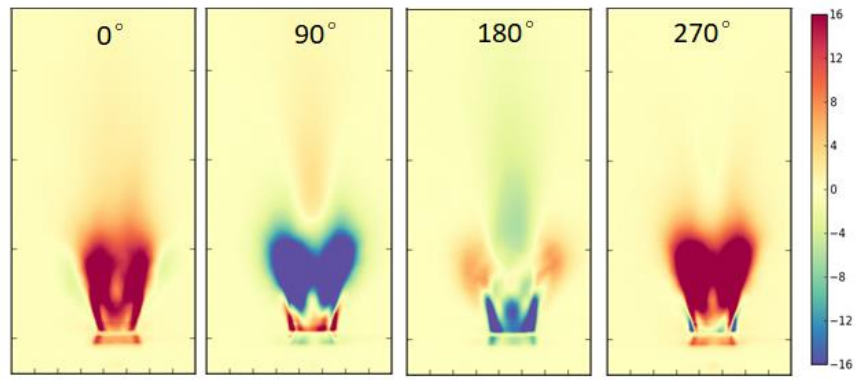

(a) Flame

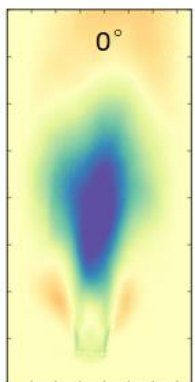

$90^{\circ}$
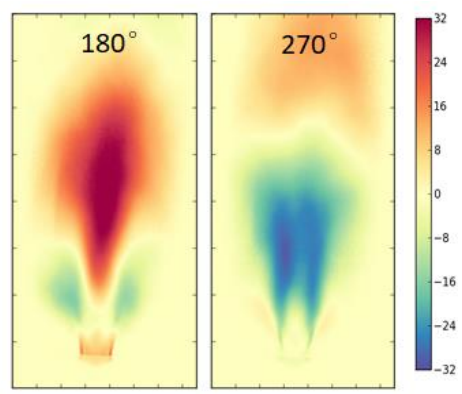

(b) Hot burnt gas

Fig.16 DMD mode $(f=32 \mathrm{~Hz})$ for Case 5

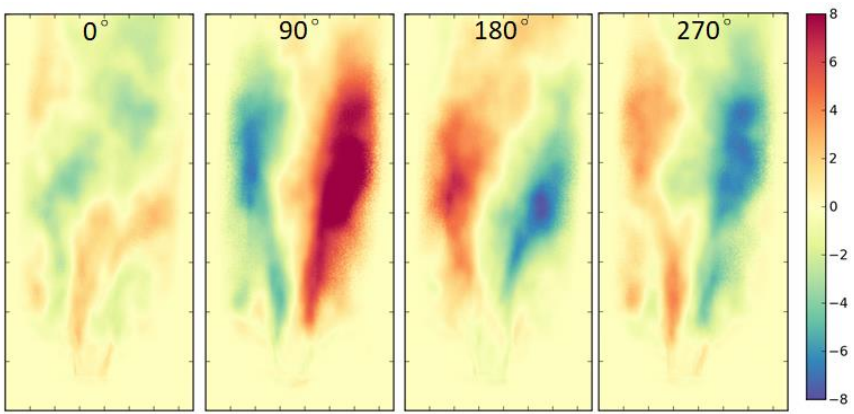

Fig.17 DMD mode $(f=7 \mathrm{~Hz})$ of hot burnt gas for Case 5

Fig. 16 presents the DMD mode phases of flame and hot burnt gas for Case 5. The mode phases of flame present a process of downstream to upstream development, which is the typical feature of LF mode. Meanwhile, the mode phases of hot burnt gas present a process of upstream to downstream development. The combustion instability is in LF mode, which is corresponding to the longitudinal mode. Meanwhile,
Fig. 17 is the mode phases of the special frequency $\left(f_{\mathrm{b}}\right)$. This special frequency is $6 \mathrm{~Hz}$ and $7 \mathrm{~Hz}$ for Case 4 and Case 5, respectively. This mode is different from the longitudinal mode presented in Fig.17. This special mode may be due to the flicker of the hot burnt gas caused by buoyancy.

\section{CONCLUSIONS}

An experimental platform of propane/air premixed swirling combustion was developed. Dynamical characteristics of combustion instability were investigated experimentally, mainly focusing on the unstable modes and the features of downstream hot burnt gas. The imaging sequences of swirling flame were investigated by DMD method. The main conclusions are summarized below:

1. The stability map is obtained through experiments. Three typical unstable modes (LF mode, $\mathrm{C}_{1 / 4}$ mode and $\mathrm{P}_{1 / 2}$ mode) are detected, which are well consistent with the resonant acoustic mode of combustor system. A mixed mode is found as a transition between LF mode and $\mathrm{C}_{1 / 4}$ mode. The transition is mainly related to the combustion power and equivalence ratio.

2. DMD method can decompose complex combustion oscillation modes effectively and distinguish various unstable mode features. The LF mode and $\mathrm{C}_{1 / 4}$ mode are both longitudinal modes, which exhibit different process in mode phase. The DMD spectrums of flame are consistent with the PSD spectrums of $\mathrm{OH}^{*}$ and acoustic signals in frequency domain. Thus, there is a coupling between heat release and acoustic pressure.

3. Simultaneous imaging in the UV-IR-cut and Near-IRpass bands has been successfully applied to the diagnosis of combustion oscillation. The downstream hot burnt gas in stable and unstable combustion show significant different behaviour. For unstable modes, the intrinsic frequencies of the hot burnt gas are similar to the flame. Though there is strong coupling, a time lag is found in time space. Meanwhile, a special mode $(7 \mathrm{~Hz})$ is discovered in the hot burnt gas, suggesting the dynamics of hot burnt gas is different from the flame. The specific coupling process between flame and hot burnt gas may need further study. However, the simultaneous imaging in the visible and nearinfrared bands provides a new idea for the investigation of combustion instability.

\section{ACKNOWLEDGMENTS}

The present work is financially supported by the Natural Science Foundation of China (No. 91541108) and the Fundamental Research Funds for the Central Universities (No. 2017FZA4032 and 2017XZZX008-04). The authors greatly thank Dr. Daniel Durox, Dr. Kevin Prieur and Prof. Sebastien Candel from Laboratory EM2C, CentraleSupelec, for their kindest helps and enlightening comments to this work.

\section{ABBREVIATES:}

$C_{1 / 4}$ mode

$D M D$
Combustor $1 / 4$ wave mode Dynamical Mode Decomposition 


$\begin{array}{ll}F F T & \text { Fast Fourier Transform } \\ L F \text { mode } & \text { Low Frequency mode } \\ P_{1 / 2} \text { mode } & \text { Plenum 1/2 wave mode } \\ P M T & \text { Photomultiplier } \\ P S D & \text { Power Spectrum Density } \\ R M S & \text { Root Mean Square }\end{array}$

\section{REFERENCES}

Culick, F. E. C., and P.Kuentzmann, 2006, "Unsteady Motions in Combustion Chambers for Propulsion System," NATO Research and Technology Organization.

Guo, Z. H., Wang, S., Li, L., Zhang, C. Y., and Sun, X., F, 2009, "Experimental study on combustion instability of lean premixed swirling flame," Journal of Aerospace Power. (in Chinese), 24(12), pp. 2367-2642.

Huang, Y., and Yang, V., 2004, "Bifurcation of flame structure in a lean-premixed swirl-stabilized combustor: transition from stable to unstable flame," Combustion and Flame, 136(3), pp. 383-389.

Huang, Y., Wang, S., and Yang, V., 2012, "Systematic Analysis of Lean-Premixed Swirl-Stabilized Combustion," AIAA Journal, 44(44), pp. 724-740.

Kim, K. T., Lee, J. G., Lee, H. J., Quay, B. D., Santavicca, and Domenic, "Characterization of Forced Flame Response of Swirl-Stabilized Turbulent Lean-Premixed Flames in a Gas Combustor," pp. 823-831.

Li, J., Durox, D., Richecoeur, F., and Schuller, T., 2015, "Analysis of chemiluminescence, density and heat release rate fluctuations in acoustically perturbed laminar premixed flames," Combustion and Flame, 162(10), pp. 3934-3945.

Min, C. L., Yoon, J., Joo, S., Kim, J., Hwang, J., and Yoon, Y., 2015, "Investigation into the cause of high multimode combustion instability of $\mathrm{H} 2 / \mathrm{CO} / \mathrm{CH} 4$ syngas in a partially premixed gas turbine model combustor," Proceedings of the Combustion Institute, 35(3), pp. 32633271.

Palies, P., Durox, D., Schuller, T., and Candel, S., 2010, "The combined dynamics of swirler and turbulent premixed swirling flames," Combustion and Flame, 157(9), pp. 16981717.

Pankiewitz, C., and Sattelmayer, T., 2003, "Time Domain Simulation of Combustion Instabilities in Annular Combustors," Journal of Engineering for Gas Turbines and Power, 125(3), pp. 391-394.

Schmid, P., and Sesterhenn, J., 2010, "Dynamic Mode Decomposition of numerical and experimental data," Journal of Fluid Mechanics, 656(10), pp. 5-28.

Schuller, T., Durox, D., and Candel, S., 2002, "Dynamics of and noise radiated by a perturbed impinging premixed jet flame," Combustion and Flame, 128(1-2), pp. 88-110.

Schuller, T., Durox, D., and Candel, S., 2003, "Selfinduced combustion oscillations of laminar premixed flames stabilized on annular burners," Combustion and Flame, 135(4), pp. 525-537.

Selle, L., Benoit, L., Poinsot, T., Nicoud, F., and Krebs, W., 2006, "Joint use of compressible large-eddy simulation and Helmholtz solvers for the analysis of rotating modes in an industrial swirled burner," Combustion and Flame, 145(1), pp. 194-205.

Weigand, P., Meier, W., Duan, X. R., Stricker, W., and Aigner, M., 2006, "Investigations of swirl flames in a gas turbine model combustor : I. Flow field, structures, temperature, and species distributions," Combustion and Flame, 144(1), pp. 225-236. 